This is a post-print version of a manuscript that has been accepted for publication in the Journal of Medical Internet Research (JMIR) Mental Health on 23 August 2021. Please consult the article in published format when available.

\title{
Opening the Black Box of Daily Life in Non-Suicidal Self-Injury Research: With Great Opportunity Comes Great Responsibility
}

\author{
Glenn Kiekens ${ }^{1,2}$, Kealagh Robinson ${ }^{3}$, Ruth Tatnell ${ }^{4}$, \& Olivia J. Kirtley ${ }^{2}$ \\ ${ }^{1}$ Faculty of Psychology and Educational Sciences, Clinical Psychology, KU Leuven, Leuven, \\ Belgium. \\ ${ }^{2}$ Department of Neurosciences, Center for Contextual Psychiatry, KU Leuven, Leuven, Belgium. \\ ${ }^{3}$ School of Psychology, Te Herenga Waka-Victoria University of Wellington, Wellington, New \\ Zealand. \\ ${ }^{4}$ Faculty of Health, School of Psychology, Deakin University, Melbourne, Australia. \\ ORCID IDs: GK: 0000-0001-8747-3385, KR: 0000-0002-9367-7445, RT: 0000-0001-6068-9864, \\ OJK: 0000-0001-5879-4120
}

\section{Funding}

This research was supported by Postdoctoral Fellowships from the Research Foundation Flanders awarded to the first and last author [12ZZM21N (GK), 1257821N (OJK)] and by the Royal Society of New Zealand Marsden Fund [18-VUW-162] awarded to the second author (KR).

\section{Disclosures/Conflicts of Interest}

OJK reports grants from UCB Community Health Fund, outside the submitted work. The other authors have no competing interests to declare.

Correspondence concerning this article should be addressed to Glenn Kiekens, KU Leuven, Faculty of Psychology and Educational Sciences, Clinical Psychology, Tiensestraat 102, box 3720, 3000 Leuven, Belgium

E-mail: Glenn.Kiekens@kuleuven.be 


\begin{abstract}
Although non-suicidal self-injury (NSSI) - deliberate damaging of body tissue without suicidal intent - is a behavior that occurs in interaction with real-world contexts, studying NSSI in the natural environment has historically been impossible. Recent advances in real-time monitoring technologies have revolutionized our ability to do exactly that, providing myriad research and clinical practice opportunities. In this vision paper, we review new research pathways to improve our ability to understand, predict and prevent NSSI, and provide critical perspectives on the responsibilities inherent to conducting real-time monitoring studies on NSSI. Real-time monitoring brings unique opportunities to advance scientific understanding about: (I) the dynamic course of NSSI, (II) the realtime predictors thereof and ability to detect acute risk, (III) the ecological validity of theoretical models, (IV) the functional mechanisms and outcomes of NSSI, and (V) the promotion of personcentered care and novel technology-based interventions. By considering the opportunities of real-time monitoring research in the context of the accompanying responsibilities (e.g., inclusive recruitment, sound and transparent research practices, participant safety and engagement, measurement reactivity, and researcher well-being and training), we provide novel insights and resources to open the black box of daily life in the next decade(s) of NSSI research.
\end{abstract}

Keywords: real-time monitoring, non-suicidal self-injury, NSSI, experience sampling, ecological momentary assessment, digital psychiatry 


\section{Introduction}

Non-suicidal self-injury (NSSI), defined as the direct and deliberate damage of one's body tissue without suicidal intent [e.g., cutting and hitting oneself; 1], is a behavior seemingly at odds with the principles of minimizing pain and maximizing pleasure, which guide most human behaviors. One in five people engage in NSSI at least once before the age of 25 [2,3], and doing so increases their risk for future suicidal thoughts and behaviors and mental health conditions [4, 5], and other adverse developmental outcomes [6-8]. Unfortunately, few individuals access support for their NSSI [9], with many young people who self-injure not finding their way to treatment [10]. Together, these findings highlight NSSI as behavior that warrants greater awareness and a better understanding. A viewpoint that the American Psychiatric Association formally emphasized with the inclusion of NSSI as a "condition requiring further study" in the DSM-5 [11].

Taking stock of the research published in the past decade reveals substantial advances in our understanding of the epidemiology, phenomenology, and developmental course of NSSI [2, 3, 10, 12]. Longitudinal cohort studies have substantially advanced knowledge regarding intra- and interpersonal risk and protective factors that clarify who is at the highest risk for developing $[13,14]$ and/or continuing NSSI behavior during adolescence and emerging adulthood $[10,15,16]$. Unfortunately, our understanding of when young people are at risk of NSSI in everyday life has not progressed similarly. We see three main reasons hindering this knowledge progression. First, it is an ecological fallacy to believe that a nomothetic approach that provides between-group knowledge about who is relatively at high risk throughout adolescence and emerging adulthood can be translated to the here and now at the individual level $[17,18]$. Indeed, knowing that someone is developmentally at risk to engage in NSSI [e.g., due to a history of victimization; 19] tells us little about when that person is most likely to selfinjure in everyday life. Second, nearly all longitudinal research studies used observation windows from months-to-years to clarify developmental risk [20]. However, retrospectively aggregating data over months-to-years (e.g., "Have you self-injured since last year?") lacks the temporal precision to detect individual risk within minutes-to-hours. Third, and perhaps most importantly, researchers have historically been constrained by practical restrictions that rendered frequent assessments of NSSI in the daily lives of those with lived experience virtually impossible. Nevertheless, in a new era of precision medicine, if we are to enable individualized intervention when and where it is most needed, then research needs to take an idiographic approach in which risk stratification repeatedly occurs in the natural environment with individuals serving as their own control [18]. 


\section{Out of the laboratory and into everyday life}

Recent advances in digital technology now make it possible for researchers to take such an idiographic approach, shifting research from the laboratory into the everyday environment where NSSI thoughts, urges, and behaviors occur. Real-time monitoring (also called experience sampling or ecological momentary assessment) is a structured self-report diary technique in which individuals provide information on their situational context, feelings, thoughts, and behavioral patterns in the flow of daily life [21, 22]. Self-report questionnaires are completed multiple times throughout the day for several days or weeks. Participants are prompted to fill in questionnaires either during predetermined intervals of time (e.g., every 2 hours; interval-contingent sampling), at random unpredictable moments (i.e., signal-contingent sampling), or following an event of interest [i.e., event-contingent sampling; 23]. Daily diaries are a particular case of interval-contingent sampling in which assessments occur only once and typically at the end of each day. Real-time monitoring methods are not a new methodology [24], with roots in ecological psychology, which argues that behavior can only be understood when investigated in the context in which it occurs [25]. Although initial real-time monitoring studies of NSSI relied upon pagers and personal digital assistants [26, 27], the ubiquity of mobile smartphones in today's society [28] has made it practically feasible for the broader research community to study NSSI and its contextual determinants in everyday life. The increased practicality of real-time monitoring methods offers a promising avenue to answer critical questions and engage researchers and clinicians in collaborative discussions. However, real-time monitoring methods, which focus on NSSI, also present significant ethical and practical challenges.

Given that real-time monitoring research is burgeoning [for recent reviews, see 29, 30, 31], it is timely to consider the valuable new directions in which the field could be heading when studying NSSI outside the laboratory, in everyday life. In 2019, the International Society for the Study of SelfInjury established a Consortium for Research on Self-Injury in Everyday Life to help build expertise and capacity in a rapidly growing field [32]. In this vision paper, arising from the work of the Consortium, we review new research pathways using real-time monitoring methods to improve our ability to understand, predict and prevent NSSI, and provide critical perspectives on the responsibilities inherent to conducting real-time monitoring studies on NSSI. In doing so, we identified critical open questions that require further investigation and offer guidance and concrete recommendations for future studies. 


\section{Opening the black box of daily life brings exciting new opportunities for science and practice}

In this section, we outline five key opportunities that real-time monitoring provides for advancing our ability to understand, predict, and prevent NSSI thoughts, urges, and behaviors in the lives of those at risk (Table 1).

\section{[INSERT TABLE 1 ABOUT HERE]}

Opportunity 1: A better understanding of the short-term course of NSSI thoughts, urges, and behavior through direct observation and precise measurement

Real-time monitoring enables rigorous descriptive research about the course of NSSI thoughts, urges, and behaviors. Initial work has demonstrated that NSSI thoughts frequently occur among individuals who self-injure but are usually short-lived and of moderate-intensity [27, 33, 34]. The propensity to experience intense and persistent NSSI thoughts has been found to increase throughout the day [34], with thought intensity fluctuating considerably from hour-to-hour for some individuals [35]. However, future work is needed to replicate these findings and many questions remain, including: dynamics over even shorter timescales (i.e., within seconds, minutes), whether different thought profiles can be identified in terms of intensity, duration, controllability, and persistence, and the degree to which changes in dynamic thought patterns relate to urges and behaviors.

Different qualitative aspects of NSSI thoughts and urges may combine to increase risk, such that the likelihood of NSSI behavior may increase in situations characterized by more intense persistent thoughts [33]. In this respect, real-time monitoring offers the opportunity to capture a fastmoving thought-to-action process through precise measurement in real-time. Research suggests that it typically takes people between 1 to 30 minutes to transition from NSSI thoughts to behavior [27, 33], meaning that, in most instances, there is a brief window of opportunity to intervene and interrupt the transition from thoughts to behavioral action. Better characterization of the thought profiles and behavioral patterns of NSSI, as well as the extent to which these can change both within and across individuals, are an essential first step in identifying individual-level predictors for risk screening and preventive intervention. 
Opportunity 2: Advancing knowledge of individual-level predictors of NSSI thoughts, urges, and behavior, and the ability to accurately detect idiographic risk

Daily life research provides a contextualized understanding of the momentary factors that explain variability in the short-term course of NSSI. Using real-time monitoring, researchers can study theoretically relevant situational, emotional, and cognitive factors to advance knowledge of individuallevel predictors for developing NSSI thoughts, NSSI urges, and for making the transition to behavior. Initial findings suggest that likelihood of these outcomes may increase when people are alone [27], after negative social appraisals and perceived conflict [36,37], or following increased negative and decreased positive affect $[35,38]$. Studies have also observed an increased risk of NSSI thoughts, urges, and behavior in the presence of high self-critical and negative repetitive thinking $[39,40]$ or low momentary self-efficacy to resist NSSI [35]. Despite this knowledge, future research is needed to clarify the relative importance of these situational, emotional, and cognitive factors at each stage of the NSSI process and their specificity in predicting NSSI compared to co-occurring behaviors [e.g., eating-disordered behaviors, suicidal thoughts; 27, 41]. Worth mentioning in this context, real-time monitoring also provides a unique opportunity to clarify the relationship with these comorbid behaviors in daily life [e.g., 42], thereby offering meaningful information to further diagnostic understanding of NSSI. Finally, the timescale on which factors exert an effect, and how their interplay can be understood mathematically (i.e., linear or non-linear effects), warrants further clarification.

Building upon empirically-derived answers to these critical questions, the next fundamental step is developing risk prediction models that can accurately detect when someone is at imminent risk for engaging in NSSI. Using each individual's longitudinal data, researchers can select and combine risk and protective factors to create risk stratification indices of NSSI thoughts, urges, and behavior in the natural environment for a particular person [e.g., 43 in the case of smoking behavior]. Statistical classification approaches (also known as machine learning) and validation techniques can be employed to identify the most suitable person-specific combination of risk factors [44, 45]. However, two caveats should be acknowledged for future research in this area. First, although idiographic risk prediction models will scale up the ability to identify individuals at acute risk for NSSI thoughts, urges and behavior in daily life, making better use of mobile technologies' growing capacities will be pivotal not to leave individuals identified as at-risk without the necessary support (see opportunity 5). Second, because real-time monitoring for prolonged periods becomes burdensome, it will be crucial to capitalize on the ever-growing technological capacities and explore the utility and integration of passively-collected information in these models [46]. For example, smartphones continuously track a 
wealth of 'in the moment' information (e.g., call or SMS logs, location recording), and initial investigation supports the feasibility of using wearables to measure psychophysiology among high-risk adolescents [47]. Importantly, these two caveats illustrate that real-time monitoring of NSSI thoughts and behaviors also brings considerable ethical, legal, and practical challenges regarding inclusivity, informed consent, and participant safety and burden (see responsibilities section for a discussion of these challenges).

\section{Opportunity 3: Test existing theories and develop new models that bridge the idiographic and} nomothetic divide and explain who is at risk, and when

Real-time monitoring provides researchers with the opportunity to put existing theories to the test in daily life. Contemporary theories of NSSI posit that the joint influence of social, affective, and cognitive vulnerabilities cause risk for NSSI via idiographic micro-processes that play out in the realm of ordinary life. However, these psychological processes are typically evaluated using cross-sectional and traditional longitudinal surveys - designs that do not have the necessary temporal granularity or ecological validity to reliably assess these theories' dynamic real-life components. Real-time monitoring overcomes this limitation. For instance, Hughes and colleagues observed that momentary negative affect and repetitive negative thinking synergistically predict NSSI in daily life, thereby providing evidence for the Emotional Cascade Model [48]. A limitation of this model is that it does not address why someone chooses to engage in NSSI instead of other dysregulated behaviors. In this respect, the Benefits and Barriers Model argues for a unique role of self-criticism in developing NSSI [49], whereas the Cognitive-Emotional Model argues for an expanded role of NSSI-specific cognitions [50]. Consistent with the Cognitive-Emotional Model, cross-sectional evidence suggests that behaviorspecific beliefs (e.g., self-efficacy to resist NSSI) explain why individuals use NSSI instead of risky alcohol use, or disordered eating, when distressed [51]. Investigations of these models in daily life are currently ongoing $[35,39,52]$. Emerging evidence, for instance, suggests that momentary belief in one's ability to resist NSSI is a robust short-term predictor of NSSI behavior among young adults in daily life [35]. However, more work is required to replicate and extend initial findings, including whether behavior-specific beliefs can explain engagement across different behaviors-NSSI and nonNSSI-for everyone.

Notably, existing theories of NSSI do not explicitly differentiate nomothetic and idiographic risk processes, thereby implicitly assuming that what causes risk is the same across individuals. Nevertheless, we can expect that variation in risk processes will be the rule rather than the exception 
$[17,53]$. As in most psychology areas [54], existing models are verbal theories, which formulate a narrative of how NSSI behavior manifests rather than translating the theory's tenets and assumptions into a formal model using mathematical notation. Emerging work underscores the need for novel models in psychology to make formal predictions [54-56], which would allow researchers to precisely estimate what a theory predicts at different measurement levels in computational models, and compare this with real-world data. Real-time monitoring can facilitate the generation of theoretical models that conceptualize NSSI as a complex system of contextualized dynamic processes. When formalized, dynamic and contextually-informed theories could predict concretely and precisely when NSSI thoughts, urges, and behaviors are likely to occur, and for whom. Such theory construction would progress understanding of factors that increase/decrease the risk for everyone, a subgroup of individuals, or a specific individual [57], and help overcome the research-practice gap by allowing practitioners to consider what causes risk for an individual while still enabling the scalability and generalizability of these predictions to be evaluated [58].

Opportunity 4: Insight into the functional mechanisms, day-to-day outcomes, and relationship of dynamic patterns with meaningful longer-term developmental changes and outcomes

By providing the opportunity to track the dynamic processes in the moments that lead up to and follow self-injurious behavior, real-time monitoring allows investigation of the functional mechanisms that maintain NSSI in daily life. According to the Four-Function Model [59], NSSI may be used to mitigate negative or unwanted thoughts and feelings (i.e., intrapersonal negative reinforcement), to generate emotion as a form of stimulation (i.e., intrapersonal positive reinforcement), to escape from uncomfortable social situations (i.e., interpersonal negative reinforcement), or to seek support from others (i.e., interpersonal positive reinforcement). Although the Four-Function Model has received considerable empirical support in cross-sectional studies [for reviews and meta-analytic findings see 12,60$]$, longitudinal measurement in real-life is needed to model the temporal contingencies of interpersonal and intrapersonal processes. A recent review of daily life studies of NSSI revealed the most evidence for intrapersonal negative reinforcement but also found substantial inconsistencies [30]. For example, although some studies observed an increase in negative affect before and a decrease following NSSI behavior [e.g., 61], others failed to replicate this pattern [e.g., 62], and some even found increased, rather than decreased, negative affect following NSSI behavior [e.g., 63]. Although investigations of the other reinforcement processes are scarce, findings were also mixed [30]. An important recommendation for future work is to consider 
timeframes more carefully. Real-time monitoring studies that add brief follow-up surveys to their protocol when people report momentary NSSI thoughts and urges provide a unique opportunity to unravel contingencies that unfold across shorter (i.e., seconds and minutes) and longer (e.g., hours and days) time intervals [64].

Apart from providing insight into the functional mechanisms, such studies would also clarify the psychosocial outcomes of NSSI in daily life. For example, engagement in NSSI may lead to interpersonal conflict as well as increased social support [65-67], feelings of shame [68], and experiencing stigma (especially when scars are visible) $[69,70]$ which may, in turn, increase social withdrawal and the likelihood of future NSSI. Hence, much could be learned from future investigations that adopt a transactional framework in which NSSI outcomes and psychosocial experiences might influence each other reciprocally in daily life. Incorporating real-time monitoring within prospective cohort studies [i.e., measurement burst designs; 71] can uniquely inform how shortterm patterns relate to longer-term developmental change and outcomes. Although already employed in depression and substance use research $[72,73]$, these measurement burst designs are an untapped resource for NSSI research. For instance, the degree to which NSSI thoughts are self-sustaining in daily life (i.e., auto-correlation) could signal a more challenging recovery process [74] or help explain why some individuals (e.g., those with depression) are at risk of a more chronic NSSI course [75]. Given the relationship between NSSI and suicidal thoughts and behaviors throughout development [4, 76], a critical question is clarifying whether a dynamic blueprint of NSSI can help gauge future risk of suicidal forms of self-injury. Providing greater clarity regarding potential day-to-day and longer-term developmental outcomes would aid scientific understanding and provide valuable information for prevention efforts and clinical risk assessment.

\section{Opportunity 5: Promotion of person-centered care, personalized prevention, and novel technology-based interventions}

Over the last decades, mental health care has gradually shifted from hospital-based to community-based care, and changed focus from symptom reduction to patient-defined recovery [21]. Through repeated observation of emotions, thoughts, symptoms, NSSI outcomes, and contextual determinants thereof in patients' lives, real-time monitoring can help respond to the call for more person-centered care in the treatment of NSSI [74]. For instance, through easy to interpret visualizations of real-time monitoring data, information on individual functioning and patient-defined outcomes can be fed back into the therapy room. This way, real-time monitoring could facilitate 
psychoeducation about relevant processes - that patients may otherwise be unware of - and give clinicians and patients a valuable tool to monitor and tailor treatment according to patients' dynamic therapy needs. For an example of such a real-time monitoring tool, see the KU Leuven m-Path app and platform. However, to enable the use of real-time monitoring as a therapeutic tool in the treatment of NSSI, pilot studies are required to address the barriers [e.g., burden and fear of reactivity; 77] and requirements for successful implementation [e.g., availability of an accessible and reliable platform; 78] of real-time monitoring as a blended-care tool. Building upon this, randomized control trials are needed to determine how, when (e.g., unguided in the moment or guided during a clinical session), and which type of feedback (e.g., overall functioning, activities, and social interactions or NSSI-specific triggers and risk processes) should be offered. Co-developing answers to these open questions with all stakeholders involved (i.e., people with lived experience, researchers, clinicians, software developers) represents a critical step to harness the potential of real-time monitoring for NSSI treatment.

Finally, real-time monitoring provides scientist-practitioners the opportunity not only to observe but also to deliver support in people's everyday lives, taking mental health care beyond the clinical setting and into daily life. Ecological Momentary Interventions (EMIs) are delivered in realtime through a smartphone app or wearable (e.g., smartwatch) and can be offered as a self-help mHealth intervention or to augment and extend the reach of existing treatments [21, 79]. Initial findings indicate the acceptability and potential of EMIs and mobile apps that target NSSI [80-82], but this remains a largely underexplored area of research. A sophisticated EMI which currently shows promising results in mental health research is Just-In-Time Adaptive Interventions [JITAIs; 83], which could help people resist the urge to self-injure when needed most in daily life. JITAIs tailor interventions to the risk status (e.g., low, medium, high) and receptivity of people within the environmental context, thereby enabling timely and contextually informed interventions for behaviors that are highly dynamic [84]. Give these possibilities, the use of JITAIs is already emerging in suicide research [85], with similar efforts needed to develop, evaluate, and integrate these new treatment methods into a stepped-care model for NSSI.

\section{Summary}

Opening the black box of daily life in NSSI research has considerable potential to advance scientific understanding about: (1) the short-term course of NSSI thoughts, urges, and behavior; (2) the individual-level predictors thereof and ability to accurately detect imminent risk; (3) the ecological validity of theoretical models and the possibility to explain when NSSI thoughts and behaviors are 
most likely to occur and for whom; (4) the functional mechanisms of NSSI and relationship of dynamic patterns with day-to-day and meaningful longer-term change and outcomes, and; (5) the implementation of real-time monitoring to prevent key NSSI and support individuals in distress when they need it most. However, studying NSSI 'in the wild,' outside a controlled laboratory environment, also presents unique challenges for which there are no established gold-standard solutions.

\section{With great opportunity comes great responsibility}

In the following section, we outline vital responsibilities when planning and carrying out realtime monitoring research of NSSI thoughts and behaviors (Table 2). Although some considerations are universally applicable to real-time monitoring research, here we focus on the issues, which have particular relevance in the context of NSSI and point to open questions in these domains for future research.

\section{[INSERT TABLE 2 ABOUT HERE]}

\section{Responsibility 1: Recruitment and inclusivity}

When the goal is to understand the dynamic course of NSSI, sample diversity, without becoming tokenistic, should be prioritized to safeguard against falsely generalizing from one individual's (or a subgroup's) experience to the entire population. Therefore, we recommend actively engaging with members of more vulnerable groups, where the risk of NSSI and suicide may be higher than in the general population [e.g., LGBTQIA+, black, indigenous, and other people of color (BIPOC), and people facing homelessness; 86, 87], and utilizing their input on how best to approach the research. Inclusive research is always essential [88], but especially when dealing with sensitive topics such as NSSI. For instance, working with people from different cultural and linguistic backgrounds requires flexibility in the way themes such as NSSI, suicide, and death are considered and discussed due to differences in cultural norms and language use $[89,90]$. Some people may also not have access to a smartphone with a $4 \mathrm{G}$ connection or might share one smartphone in a household or family, conferring additional privacy concerns $[91,92]$. Therefore, researchers might aim for a budget that allows devices or data bundles to be provided to participants who need them, rather than excluding them. Importantly, however, if the real-time monitoring protocol involves deploying EMIs, researchers should be aware that participants may have come to rely upon the device and the EMI during the study period and that withdrawing these at the end of the study may leave participants without crucial support. Flexible compensation schedules, in which participants can choose to keep the smartphone as compensation for their participation, may be one solution. If practically unfeasible- 
either because of logistical constraints on the researchers or because the EMI requires a mobile data plan that participants cannot access - participants should not be left without support and could be offered alternative interventions (not requiring mobile data access) following the completion of the study.

When planning to recruit school-aged individuals to real-time monitoring studies, extra consideration should be given to data collection within the school context. For instance, schools may prohibit access to devices during the school day. van Roekel and colleagues [93] provide useful recommendations for working in school contexts, such as ensuring that there is a strong alliance with schools, teachers, and parents, using participation cards so students are allowed to use their phones when prompted, and making sure that schools also benefit from the research. Having a specific person who is the 'face' of the study within the school can also be useful [for a detailed discussion of the challenges of conducting NSSI research generally within schools, see 94].

\section{Responsibility 2: Informed consent and participant briefing}

Given that real-time monitoring research takes place in daily life without the researcher being present, additional consideration of the informed consent process and participant briefing is needed [95]. Information regarding the study's often intensive nature, such as the study's time course, the number of surveys per day, and the periods during which participants can expect prompts, should be made clear to potential participants before study enrollment. Given that participant compliance rates in real-time monitoring research can vary [96], participant briefing should cover whether financial compensation or other benefits of research participation are compliance-dependent and, if so, how many reminders will be sent. When studying NSSI, in particular, it is paramount that participants are informed about the safety procedures (especially when this involves human-led intervention contingent upon a survey response) and potential consequences of these safety procedures (e.g., when will the duty of care override the confidentiality principle and who will then be informed). The informed consent process should also clarify whether additional data will be passively collected (e.g., location coordinates, incoming and outgoing SMS messages and calls, app usage statistics, accelerometer data, etc.) and make participants aware of the detailed level of data that can be collected without their active engagement. Poor digital literacy may threaten adequate informed consent [97], especially for passively collected data. Jacobson and colleagues [95] provide several valuable suggestions to ensure that participants have a complete and detailed understanding of the study, such as highlighting essential information, using comprehension quizzes, and preventing participants from 
scrolling through the informed consent without reading it (when provided online). Considering the extensive amount of information participants receive, it could be worthwhile to request consent for each part of the study separately (e.g., data collection schedule, intervention component, safety protocols/plan). Providing real-world examples utilizing interactive videos or apps that can read information aloud could also be used to facilitate comprehension and mitigate the risk of poor digital literacy.

When working with minors, both the young person's assent and informed consent from their parent or caregiver will typically be required. However, as a highly stigmatized behavior, NSSI is often hidden from others [98]. Although disclosure to parents and caregivers can facilitate helpseeking and improve coping, it can also negatively impact the parent-child relationship and the wider family system $[66,99]$ and lead the young person to worry about the involvement of parents or caregivers [100]. Although parents will often be informed when recruiting young people within a clinical setting, we recommend explaining the study's purpose in general (e.g., "to study interactions, emotions, thoughts, and behavior in daily life") rather than following NSSI-specific terms to avoid forced disclosures. This framing method also means that individuals may avoid reflecting upon their participation through a disease-perspective or NSSI-labeled identity [101, 102]

\section{Responsibility 3: Participant safety and risk-monitoring}

In real-time monitoring studies of suicidal thoughts and behaviors, ethical considerations regarding participant safety are, justifiably, a recurring concern [95, 103]. In contrast, very few NSSI real-time monitoring studies report procedures for safeguarding and supporting participants during the study [100]. This may be because high suicide risk is sometimes an exclusion criteria for participation [e.g., 33, 104, 105], and participant safety procedures are generally to safeguard participants at high or imminent risk of making a suicide attempt [27, 38]. Real-time monitoring studies tread a fine line between research and intervention and there must be a "goodness of fit" between a study's objectives and the design of the safety procedures [100]. For example, in a study of NSSI behavior, contacting participants every time they report engaging in NSSI would defeat the study's purpose and may even discourage participants from reporting NSSI during the study period [100]. If a participant scores highly on a momentary measure of suicidal intent, contacting the participant may be appropriate and would not compromise the study's goal of assessing NSSI. A critical ethical issue underlying participant safety procedures is that even though an increase in suicidal intent is unlikely to be caused by study participation [106, 107], the individual's status as a participant in a real-time monitoring 
study creates an opportunity for intervention that would otherwise not exist. Therefore, we recommend assessing suicidal intent in real-time monitoring research on NSSI thoughts and behaviors and advise against the exclusion of people at risk for suicide.

The first consensus statement on ethical and safety procedures for real-time monitoring studies with individuals at risk of suicide recently emerged [108]. The recommendations include collecting contact information for participants and a close contact, completing a safety plan at study enrollment, monitoring responses at least once per day, and in the event of a participant being at imminent risk of suicide, for a researcher to contact them. Interestingly, our experiences have been somewhat different, with clinicians expressing concern that a researcher may be the "first-responder" to a suicidal crisis. In this regard, contacting the participant's clinician may be better. However, no consensus was reached regarding whether the researcher should contact a participant's clinician in the event of high or imminent suicide risk [108]. The logistical challenges of actively monitoring participants' responses and potentially intervening should not be underestimated, especially for large studies where multiple participants may require intervention simultaneously. Ensuring that adequate staffing and resources are available to carry out the study's safety procedure is essential. In the interests of transparency and to evaluate safety procedures in real-time monitoring studies of NSSI, we recommend reporting details regarding participant safety protocols as standard. Moreover, qualitative research should substantively investigate participants' and clinicians' preferences for safety procedures concerning NSSI outcomes.

\section{Responsibility 4: Measurement reactivity}

A particular concern in all NSSI and suicide research is that asking individuals questions about their self-harm-related thoughts and behaviors may increase them. However, evidence suggests that this is not the case $[109,110]$, prospectively across young adult [111], adult and adolescent samples [112, 113], and when using various NSSI-related stimuli [images and words; 112]. In contrast, findings indicate that participants find their participation in research on NSSI and suicidal behavior to be beneficial $[109,111,113]$.

Although it appears that asking people about NSSI and suicide at a single time point has no impact on self-harm-related thoughts and behaviors, real-time monitoring requires repeated questioning on these topics. To date, no research has tested the potential iatrogenic effects of real-time monitoring research specifically for NSSI, but evidence from the suicide literature is promising. Early work by Husky and colleagues [106] used real-time monitoring to assess depression, mood, and thoughts of suicide and self-harm five times a day for one week, in four samples: people with a recent 
suicide attempt, people with a past suicide attempt, people with mood disorders but no suicidal behavior, and healthy controls. In this study, there was no reactivity to the repeated questioning about self-harming thoughts across any of the four groups. Law and colleagues [104] demonstrated similar outcomes with a longitudinal design assessing 248 adults (30\% of whom reported a borderline personality disorder diagnosis, which confers additional suicide risk). In this study, the authors found no increase in suicidal thoughts and/or behaviors during the initial 2-week data collection phase, nor at a 6-month follow-up, including for people with a borderline personality disorder diagnosis. Most recently, Coppersmith and colleagues found no association between the frequency of asking about suicidal ideation and intent in a real-time monitoring study, and the severity of suicidal thoughts over time [107]. They also observed no change in survey responses when ideation was severe, where a decrease might be expected if participants were reactive to questioning. Currently, the evidence suggests no iatrogenic effects of repeated questioning about suicide and suicide-related behaviors. Further research is required to confirm whether this pattern also holds true for NSSI and is robust across different subgroups.

\section{Responsibility 5: Balancing scientific accuracy against participant burden and ensuring the research is feasible, transparent, and safe}

An important responsibility when designing a real-time monitoring study is the selection of an appropriate sampling design (i.e., fixed, interval, [semi-]random, event-based, mixed), sampling density (i.e., number of assessments per day), sampling duration (i.e., number of days/weeks), and sample size $[45,93]$. It is crucial that the selected sample shows sufficient variability in the outcomes of interest to allow investigation of the research question in daily life [45]. For example, when the aim is to clarify the transition from NSSI thoughts to behavior, base rates of thoughts and behavior must be high enough during the real-time monitoring period. To ensure this is the case, researchers need to consider the inclusion criteria carefully (e.g., by including individuals with more than five acts of NSSI behavior in the two weeks before study onboarding). The selected protocol should allow answering the pre-specified research question(s) without unnecessarily burdening participants. Balancing these needs may mean that protocols are not interchangeable across studies - there is no "one size fits all" protocol. Researchers should be cautious about, for example, adopting sampling densities used in previous studies, as these may not be suitable for addressing different research questions. In some cases, it will be perfectly justifiable — even essential — to ask participants to complete a more intense or extended real-time monitoring protocol. However, to ensure divergence 
between protocols across studies is not arbitrary, researchers must justify their protocol [114] and communicate expectations to potential participants during study onboarding.

Compared with other research methodologies, real-time monitoring studies involve a higher workload for, and burden on, participants. Therefore, we recommend piloting the feasibility and acceptability of real-time monitoring protocols using a quality improvement procedure, where protocols are first tested extensively by members of the research team and then iteratively with a selected group of participants. In our experience, this approach requires more time and planning but safeguards participants' (and researchers') investment by allowing the protocol to be modified and optimized, if needed, in response to qualitative and quantitative feedback (e.g., the time it takes to complete the questionnaire). Emerging evidence suggests that the questionnaire length, rather than sampling frequency, is associated with increased participant burden and reduced data quality [115]. Although these findings are hopeful for researchers wanting to use more intense protocols, they also underscore the necessity for careful item conceptualization and selection. To avoid a methodological 'wild west', we advise researchers to make their items publicly available [see the Experience Sampling Method (ESM) Item Repository; 116] and report (where possible) the multilevel reliability and validity of operationalized NSSI outcomes. Against the backdrop of the replication crisis, preregistration and data sharing (open data) are also increasingly used to increase reproducibility and avoid wasting public resources [117]. These considerations are especially relevant considering the high demands of a real-time monitoring study on participants and researchers. For a tutorial and template for the preregistration of real-time monitoring studies, see Kirtley and colleagues [118]. Finally, there is consensus among researchers and clinicians that real-time monitoring platforms should be secure and compliant with the relevant privacy laws [108]. In some cases, data collected with real-time monitoring apps may be stored in another country, and thus subject to different privacy legislation from the researcher's home country. Researchers should thus be well-informed before commencing a real-time monitoring study and, when unsure, should contact their institution's research governance or data management department for clarification.

\section{Responsibility 6: Compensation and recognition of participant engagement}

Consideration should be given to distributive justice principles such that participants benefit from their engagement in the study. Prior work found that the majority of young people who take part in traditional survey research investigating sensitive topics such as NSSI report that their participation allowed them the opportunity to develop greater self-awareness and had altruistic value [113]. 
However, real-time monitoring studies tend to require more time and effort from participants. To give some initial insight into the experience and expectations of participants who self-injure, we present additional results from a recent real-time monitoring study in which emerging adults were prompted every 90 minutes during waking hours for 12 days [35].

Following the monitoring period, approximately 4 in 5 participants indicated increased selfawareness of feelings and thoughts (Table 3). About half considered the protocol demands to be tiring, but most participants also described their participation as positive. Notably, all participants reported being interested in receiving information on the key findings of the study when it concluded. Although not a primary reason for participation for most participants, they also expected feedback on their own data $(72.4 \%)$ and a monetary incentive (79.3\%). Participants considered $€ 60$ to be a fair compensation for the study protocol's demands ( 8 beeps/day for 12 days, in total 96 assessments, median compliance $79.2 \%$; IQR $=70.3-91.7 \%$ ), when they also received feedback about the overall findings and their own data. In the absence of feedback on their data, participants expected higher financial compensation for their investment. In this study, participants were reimbursed according to a structured financial scheme to encourage participation. Rather than paying a fixed amount per completed survey, a structured incentive scheme has the advantage that it allows participants to miss some surveys without direct financial consequences. For some groups (e.g., adolescents, people with low-socioeconomic status), avoiding direct financial consequences of missed surveys may be particularly important to prevent some participants from changing their daily routines to respond to each survey.

\section{[INSERT TABLE 3 ABOUT HERE]}

These findings are consistent with traditional survey research [113] and provide a first indication that real-time monitoring offers self-awareness opportunities for participants. Further, it highlights to researchers that they should actively recognize participants with lived experience of NSSI as valued partners in the research by also giving informational support. Although the majority experienced participation as positive, future work should clarify why this may not be the case for everyone so that resources can be provided to those for whom participation may have increased burden and discomfort. In this respect, it is advisable to organize a debriefing session that allows participants to share their experiences. Moreover, future work could explore the perceived meaningfulness of several strategies, such as the tailoring of sampling schedules and including a temporary 'suspend' button to offer participants flexibility and greater control over notifications [114], the randomization of 
items to reduce response fatigue [called planned missing-data designs 119, 120], and allowing for catch-up days so participants can reach the desired level of compliance [93].

\section{Responsibility 7: Researcher well-being and training}

Finally, we want to draw explicit attention to researcher well-being and training considerations. Real-time monitoring data are quite literally an individual's real-life experiences, occurring in real-time, which may lead researchers to feel especially close to participants and their experiences. Although studies have explored participants experiences of taking part in research on self-harm and suicide more broadly [121], as well as in real-time monitoring studies [122, 123], to our knowledge, no studies have investigated researchers' experiences of conducting real-time monitoring studies of NSSI or suicidal behaviors. However, the stakes are high when the research focus is on selfinjurious behavior; monitoring participants' responses for signs of imminent suicidal crisis lays great responsibility on researchers' shoulders. Where safety protocols involve routine telephone check-ins, a missed check-in may cause the researcher to fear for the participant's safety, even though there may be an innocuous explanation (e.g., the participant was driving or in the shower). Researchers may also begin to feel a high level of responsibility for participants' not engaging in NSSI or attempting suicide [i.e., feeling they are keeping the participant alive or safe; 124, 125]. Studies that employ real-time alerts when participants indicate escalating suicidal intent may lead researchers to become hypervigilant and worry that intervention may be required at any moment. Such warnings may come outside of working hours, increasing work-life balance challenges. For the researcher, all of these situations underscore a lack of controllability, which may prove highly stressful, especially when experienced over a sustained period.

Good quality training in working with individuals who engage in NSSI and suicidal behaviors is essential when conducting real-time monitoring research. Much research is carried out by doctoral students, trainees, and research assistants, who may be less experienced in collecting sensitive data and managing the accompanying emotional labor [125]. Researchers often work alone and lack a good support network [126]. Therefore, training should cover supporting a participant during an acute suicidal crisis as well as less overtly "intense" situations (e.g., where researchers are coding open text responses about participants' reasons for engaging in NSSI). A lone researcher should never carry out safety protocols involving real-time risk-monitoring and/or intervention, and responsibility should be shared among a team, whereby multiple researchers are "on-call" for a specified period. Although planning is essential, some researcher well-being challenges may only come to light once the study is 
underway [125]. Continued supervision and mentoring of researchers (of all career stages) are crucial to ensuring researcher well-being. This may involve regular debriefings with supervisors or colleagues or via external, independent counseling support. Qualitative studies with researchers who work on sensitive topics also highlight the importance of self-care and actively engaging in positive, non-workrelated activities as valuable buffers against emotional distress $[125,126]$.

\section{Summary}

Real-time monitoring technologies give researchers the practical tools to investigate NSSI thoughts, urges, and behavior as they occur, without the need for being physically present. Although this provides immense opportunities for NSSI research, real-time monitoring research is not without challenges regarding recruitment, study enrolment and planning, and participant engagement. Focusing on NSSI also creates great responsibility for privacy and data security, participant safety and risk-monitoring, and researcher well-being and training. To guide researchers who want to study the experiences of individuals who engage in NSSI, we summarize the responsibilities and ways of overcoming challenges in a functional flowchart (Figure 1).

\section{[INSERT FIGURE 1 ABOUT HERE]}

\section{Where do we go from here?}

Since Nock, Prinstein, and Sterba's seminal study in 2009 [27] which demonstrated the feasibility of studying NSSI in adolescents' everyday life, researchers now have a toolbox full of smartphone apps and wearable technology that can readily capture real-time experiences of people who engage in NSSI, in the real-world context. These advances produce a rapidly growing literature that could positively shape the field's future trajectory by facilitating a radical shift of focus from the group to the individual, from the research lab and clinic to the everyday life environment, and from traditional generalized treatment to person-centered prevention and intervention. This paper set an ambitious agenda for new research pathways to realize such a shift as we move into the next decade of NSSI research. We also offered critical perspectives on the inevitable ethical and practical challenges that come with these research pathways. In this respect, opening the black box of daily life in NSSI research is truly a double-edged sword that requires responsibility and leads to new questions. Few studies to date have specifically considered ethical issues within real-time monitoring studies of NSSI. However, these questions regarding ethical practices must be addressed with substantive empirical research, rather than being based upon the precedent of "if it ain't broke, don't fix it." In particular, research co-design and qualitative studies to capture rich information on participants' experiences of 
taking part in real-time monitoring research on NSSI offer promise. Real-time monitoring research on NSSI will advance better and more rapidly when all stakeholders' interests (i.e., individuals with lived experience, their families, researchers, and clinicians) are considered. Only by considering both the opportunities and the challenges will we be able to use real-time monitoring techniques to their full potential.

\section{Author Contributions}

All authors contributed to the conception and design of the manuscript. GK drafted the opportunity section and is responsible for the results presented in the manuscript. GK, KR, RT, and OJK drafted the responsibility section and provided critical revisions to the manuscript. All authors approved the final version of the manuscript for submission.

\section{Acknowledgments}

The authors want to thank Dr. Laurence Claes for providing comments on an earlier version of the manuscript. 


\section{References}

1. What is self-injury? International Society for the Study of Self-Injury. 2018 May. URL: https://itriples.org/about-self-injury/what-is-self-injury [accessed 2021-05-31].

2. Gandhi A, Luyckx K, Baetens I, Kiekens G, Sleuwaegen E, Berens A, et al. Age of onset of non-suicidal self-injury in Dutch-speaking adolescents and emerging adults: An event history analysis of pooled data. Compr Psychiatry. 2018 Jan;80:170-8. PMID: 29121554. doi: 10.1016/j.comppsych.2017.10.007.

3. Gillies D, Christou MA, Dixon AC, Featherston OJ, Rapti I, Garcia-Anguita A, et al. Prevalence and Characteristics of Self-Harm in Adolescents: Meta-Analyses of Community-Based Studies 1990-2015. J Am Acad Child Adolesc Psychiatry. 2018 Oct;57(10):733-41. PMID: 30274648. doi: 10.1016/j.jaac.2018.06.018.

4. Kiekens G, Hasking P, Boyes M, Claes L, Mortier P, Auerbach RP, et al. The associations between nonsuicidal self-injury and first onset suicidal thoughts and behaviors. J Affect Disord. 2018 Oct 15;239:171-9. PMID: 30014957. doi: 10.1016/j.jad.2018.06.033.

5. Wilkinson PO, Qiu T, Neufeld S, Jones PB, Goodyer IM. Sporadic and recurrent non-suicidal self-injury before age 14 and incident onset of psychiatric disorders by 17 years: prospective cohort study. $\mathrm{Br} \mathbf{J}$ Psychiatry. 2018 Apr;212(4):222-6. PMID: 29514726. doi: 10.1192/bjp.2017.45.

6. Buelens T, Luyckx K, Gandhi A, Kiekens G, Claes L. Non-Suicidal Self-Injury in Adolescence: Longitudinal Associations with Psychological Distress and Rumination. J Abnorm Child Psychol. 2019 Sep;47(9):156981. PMID: 30900112. doi: 10.1007/s10802-019-00531-8.

7. Daukantaite D, Lundh LG, Wangby-Lundh M, Clareus B, Bjarehed J, Zhou Y, et al. What happens to young adults who have engaged in self-injurious behavior as adolescents? A 10-year follow-up. Eur Child Adolesc Psychiatry. 2020 Apr. PMID: 32318877. doi: 10.1007/s00787-020-01533-4.

8. Robinson K, Garisch JA, Kingi T, Brocklesby M, O'Connell A, Langlands RL, et al. Reciprocal Risk: the Longitudinal Relationship between Emotion Regulation and Non-suicidal Self-Injury in Adolescents. Journal of Abnormal Child Psychology. 2019 Feb;47(2):325-32. PMID: 29923162. doi: 10.1007/s10802-018-04506.

9. Whitlock J, Muehlenkamp JJ, Purington A, Eckenrode J, Barreira P, Baral Abrams G, et al. Nonsuicidal selfinjury in a college population: general trends and sex differences. J Am Coll Health. 2011;59(8):691-8. PMID: 21950249. doi: 10.1080/07448481.2010.529626.

10. Steinhoff A, Ribeaud D, Kupferschmid S, Raible-Destan N, Quednow BB, Hepp U, et al. Self-injury from early adolescence to early adulthood: age-related course, recurrence, and services use in males and females from the community. Eur Child Adolesc Psychiatry. 2020 Jun. PMID: 32572615. doi: 10.1007/s00787-02001573-w.

11. American Psychiatric Association (APA). The Diagnostic and Statistical Manual of Mental Disorders, Fifth Edition. Arlington, VA: American Psychiatric Association; 2013.

12. Taylor PJ, Jomar K, Dhingra K, Forrester R, Shahmalak U, Dickson JM. A meta-analysis of the prevalence of different functions of non-suicidal self-injury. J Affect Disord. 2018 Feb;227:759-69. PMID: 29689691. doi: 10.1016/j.jad.2017.11.073.

13. Kiekens G, Hasking P, Claes L, Boyes M, Mortier P, Auerbach RP, et al. Predicting the incidence of nonsuicidal self-injury in college students. Eur Psychiatry. 2019 Jun;59:44-51. PMID: 31035219. doi: 10.1016/j.eurpsy.2019.04.002.

14. Victor SE, Hipwell AE, Stepp SD, Scott LN. Parent and peer relationships as longitudinal predictors of adolescent non-suicidal self-injury onset. Child Adolesc Psychiatry Ment Health. 2019;13:1. PMID: 30622642. doi: 10.1186/s13034-018-0261-0.

15. Kiekens G, Hasking P, Bruffaerts R, Claes L, Baetens I, Boyes M, et al. What Predicts Ongoing Nonsuicidal Self-Injury?: A Comparison Between Persistent and Ceased Self-Injury in Emerging Adults. J Nerv Ment Dis. 2017 Oct;205(10):762-70. PMID: 28817427. doi: 10.1097/NMD.0000000000000726.

16. Fox KR, Huang X, Linthicum KP, Wang SB, Franklin JC, Ribeiro JD. Model complexity improves the prediction of nonsuicidal self-injury. J Consult Clin Psychol. 2019 Aug;87(8):684-92. PMID: 31219275. doi: $10.1037 / \mathrm{ccp} 0000421$.

17. Fisher AJ, Medaglia JD, Jeronimus BF. Lack of group-to-individual generalizability is a threat to human subjects research. Proc Natl Acad Sci U S A. 2018 Jul;115(27):E6106-E15. PMID: 29915059. doi: 10.1073/pnas.1711978115.

18. Piccirillo ML, Rodebaugh TL. Foundations of idiographic methods in psychology and applications for psychotherapy. Clin Psychol Rev. 2019 Jul;71:90-100. PMID: 30665765. doi: 10.1016/j.cpr.2019.01.002. 
19. Liu RT, Scopelliti KM, Pittman SK, Zamora AS. Childhood maltreatment and non-suicidal self-injury: a systematic review and meta-analysis. Lancet Psychiatry. 2018 Jan;5(1):51-64. PMID: 29196062. doi: 10.1016/S2215-0366(17)30469-8.

20. Fox KR, Franklin JC, Ribeiro JD, Kleiman EM, Bentley KH, Nock MK. Meta-analysis of risk factors for nonsuicidal self-injury. Clin Psychol Rev. 2015 Dec;42:156-67. PMID: 26416295. doi: 10.1016/j.cpr.2015.09.002.

21. Myin-Germeys I, Kasanova Z, Vaessen T, Vachon H, Kirtley O, Viechtbauer W, et al. Experience sampling methodology in mental health research: new insights and technical developments. World Psychiatry. 2018 Jun;17(2):123-32. PMID: 29856567. doi: 10.1002/wps.20513.

22. Shiffman S, Stone AA, Hufford MR. Ecological momentary assessment. Annu Rev Clin Psychol. 2008;4:1 32. PMID: 18509902. doi: 10.1146/annurev.clinpsy.3.022806.091415.

23. Bolger N, Laurenceau JP. Intensive Longitudinal Methods: An introduction to diary and experience sampling research. New York: Guilford Press; 2013.

24. Larson R, Csikszentmihalyi M. The Experience Sampling Method. In: Reis HT, editor. New Directions for Methodology of Social and Behavioral Sciences. San Francisco: Jossey-Bass. Wiley Imprint; 1983. p. 41-56.

25. Barker RG. Ecological psychology: Concepts and methods for studying the environment of human behavior. Stanford: Stanford University Press; 1968.

26. Muehlenkamp JJ, Engel SG, Wadeson A, Crosby RD, Wonderlich SA, Simonich H, et al. Emotional states preceding and following acts of non-suicidal self-injury in bulimia nervosa patients. Behav Res Ther. 2009 Jan;47(1):83-7. PMID: 19027892. doi: 10.1016/j.brat.2008.10.011.

27. Nock MK, Prinstein MJ, Sterba SK. Revealing the form and function of self-injurious thoughts and behaviors: A real-time ecological assessment study among adolescents and young adults. J Abnorm Psychol. 2009 Nov;118(4):816-27. PMID: 19899851. doi: 10.1037/a0016948.

28. Smartphones - Statistics \& facts. Statista. 2021. URL: https://www.statista.com/topics/840/smartphones/ [accessed 2021-05-31].

29. Rodriguez-Blanco L, Carballo JJ, Baca-Garcia E. Use of Ecological Momentary Assessment (EMA) in NonSuicidal Self-Injury (NSSI): A systematic review. Psychiatry Res. 2018 May;263:212-9. PMID: 29574356. doi: 10.1016/j.psychres.2018.02.051.

30. Hepp J, Carpenter RW, Störkel LM, Schmitz SE, Schmahl C, Niedtfeld I. A systematic review of daily life studies on non-suicidal self-injury based on the four-function model. Clin. Psychol. Rev. 2020. PMID: 32949907. doi: 10.1016/j.cpr.2020.101888.

31. Gee BL, Han J, Benassi H, Batterham PJ. Suicidal thoughts, suicidal behaviours and self-harm in daily life: A systematic review of ecological momentary assessment studies. Digit Health. 2020 JanDec;6:2055207620963958. PMID: 33224516. doi: 10.1177/2055207620963958.

32. Mission Statement. International Society for the Study of Self-Injury. May. URL: https://itriples.org/ [accessed 2021-05-31].

33. Fitzpatrick S, Kranzler A, Fehling K, Lindqvist J, Selby EA. Investigating the role of the intensity and duration of self-injury thoughts in self-injury with ecological momentary assessment. Psychiatry Res. 2020 Jan 8;284:112761. PMID: 31951869. doi: 10.1016/j.psychres.2020.112761.

34. Turner BJ, Baglole JS, Chapman AL, Gratz KL. Experiencing and Resisting Nonsuicidal Self-injury Thoughts and Urges in Everyday Life. Suicide Life Threat Behav. 2019 Oct;49(5):1332-46. PMID: 30152181. doi: 10.1111/sltb.12510.

35. Kiekens G, Hasking P, Nock MK, Boyes M, Kirtley O, Bruffaerts R, et al. Fluctuations in Affective States and Self-Efficacy to Resist Non-Suicidal Self-Injury as Real-Time Predictors of Non-Suicidal Self-Injurious Thoughts and Behaviors. Front Psychiatry. 2020;11:214. PMID: 32265760. doi: 10.3389/fpsyt.2020.00214.

36. Turner BJ, Yiu A, Claes L, Muehlenkamp JJ, Chapman AL. Occurrence and co-occurrence of nonsuicidal self-injury and disordered eating in a daily diary study: Which behavior, when? Psychiatry Res. 2016 Dec 30;246:39-47. PMID: 27657802. doi: 10.1016/j.psychres.2016.09.012.

37. Victor SE, Scott LN, Stepp SD, Goldstein TR. I Want You to Want Me: Interpersonal Stress and Affective Experiences as Within-Person Predictors of Nonsuicidal Self-Injury and Suicide Urges in Daily Life. Suicide Life Threat Behav. 2019 Aug;49(4):1157-77. PMID: 30159910. doi: 10.1111/sltb.12513.

38. Andrewes HE, Hulbert C, Cotton SM, Betts J, Chanen AM. Ecological momentary assessment of nonsuicidal self-injury in youth with borderline personality disorder. Personal Disord. 2017 Oct;8(4):357-65. PMID: 27505188. doi: 10.1037/per0000205.

39. Burke TA, Fox K, Kautz M, Siegel DM, Kleiman E, Alloy LB. Real-time monitoring of the associations between self-critical and self-punishment cognitions and nonsuicidal self-injury. Behaviour Research and Therapy. 2020. PMID: 33421892. doi: 10.1016/j.brat.2020.103775.

40. Hughes CD, King AM, Kranzler A, Fehling K, Miller A, Lindqvist J, et al. Anxious and Overwhelming Affects and Repetitive Negative Thinking as Ecological Predictors of Self-Injurious Thoughts and Behaviors. 
Cognitive Ther Res. 2019 Feb;43(1):88-101. PMID: WOS:000460418300010. doi: 10.1007/s10608-01909996-9.

41. Kiekens G, Claes L. Non-Suicidal Self-Injury and Eating Disordered Behaviors: An Update on What We Do and Do Not Know. Curr Psychiatry Rep. 2020 Oct 10;22(12):68. PMID: 33037934. doi: 10.1007/s11920020-01191-y.

42. Czyz EK, Glenn CR, Arango A, Koo HJ, King CA. Short-term associations between nonsuicidal and suicidal thoughts and behaviors: A daily diary study with high-risk adolescents. J Affect Disord. 2021 Sep 1;292:33744. PMID: 34139406. doi: 10.1016/j.jad.2021.05.104.

43. Fisher AJ, Soyster PD. Generating accurate personalized predictions of future behavior: A smoking exemplar. [Preprint: https://psyarxiv.com/e24v6]. 2019.

44. Burke TA, Ammerman BA, Jacobucci R. The use of machine learning in the study of suicidal and nonsuicidal self-injurious thoughts and behaviors: A systematic review. J Affect Disord. 2019 Feb 15;245:86984. PMID: 30699872. doi: 10.1016/j.jad.2018.11.073.

45. Stange JP, Kleiman EM, Mermelstein RJ, Trull TJ. Using ambulatory assessment to measure dynamic risk processes in affective disorders. J Affect Disord. 2019 Dec 1;259:325-36. PMID: 31610996. doi: 10.1016/j.jad.2019.08.060.

46. Allen NB, Nelson BW, Brent D, Auerbach RP. Short-term prediction of suicidal thoughts and behaviors in adolescents: Can recent developments in technology and computational science provide a breakthrough? J Affect Disord. 2019 May 1;250:163-9. PMID: 30856493. doi: 10.1016/j.jad.2019.03.044.

47. Kleiman E, Millner AJ, Joyce VW, Nash CC, Buonopane RJ, Nock MK. Using Wearable Physiological Monitors With Suicidal Adolescent Inpatients: Feasibility and Acceptability Study. JMIR Mhealth Uhealth. 2019 Sep 24;0(0):e0. PMID: 31586364. doi: 10.2196/13725.

48. Selby EA, Anestis MD, Joiner TE. Understanding the relationship between emotional and behavioral dysregulation: emotional cascades. Behav Res Ther. 2008 May;46(5):593-611. PMID: 18353278. doi: 10.1016/j.brat.2008.02.002.

49. Hooley JM, Franklin JC. Why Do People Hurt Themselves? A New Conceptual Model of Nonsuicidal SelfInjury. Clinical Psychological Science. 2017;6(3):428-51. doi: 10.1177/2167702617745641.

50. Hasking P, Whitlock J, Voon D, Rose A. A cognitive-emotional model of NSSI: using emotion regulation and cognitive processes to explain why people self-injure. Cogn Emot. 2017 Dec;31(8):1543-56. PMID: 27702245. doi: $10.1080 / 02699931.2016 .1241219$.

51. Hasking P, Boyes M, Greves S. Self-efficacy and emotionally dysregulated behaviour: An exploratory test of the role of emotion regulatory and behaviour-specific beliefs. Psychiatry Res. 2018 Dec;270:335-40. PMID: 30292086. doi: 10.1016/j.psychres.2018.09.045.

52. Burke TA, Fox K, Kautz MM, Rodriguez-Seijas C, Bettis AH, Alloy LB. Self-Critical and Self-Punishment Cognitions Differentiate Those With and Without a History of Nonsuicidal Self-Injury: An Ecological Momentary Assessment Study. Behavior Therapy. 2020. PMID: 33990242. doi: 10.1016/j.beth.2020.08.006.

53. Wright AGC, Woods WC. Personalized Models of Psychopathology. Annu Rev Clin Psychol. 2020 May 7;16:49-74. PMID: 32070120. doi: 10.1146/annurev-clinpsy-102419-125032.

54. Borsboom D, van der Maas HLJ, Dalege J, Kievit RA, Haig BD. Theory Construction Methodology: A Practical Framework for Building Theories in Psychology. Perspect Psychol Sci. 2021 Feb 16:1745691620969647. PMID: 33593167. doi: 10.1177/1745691620969647.

55. Millner AJ, Robinaugh DJ, Nock MK. Advancing the Understanding of Suicide: The Need for Formal Theory and Rigorous Descriptive Research. Trends Cogn Sci. 2020 Sep;24(9):704-16. PMID: 32680678. doi: 10.1016/j.tics.2020.06.007.

56. Guest O, Martin AE. How Computational Modeling Can Force Theory Building in Psychological Science. Perspectives on Psychological Science. 2021. PMID: 33482070. doi: 10.1177/1745691620970585.

57. Lane ST, Gates KM, Pike HK, Beltz AM, Wright AGC. Uncovering general, shared, and unique temporal patterns in ambulatory assessment data. Psychol Methods. 2019 Feb;24(1):54-69. PMID: 30124300. doi: $10.1037 /$ met0000192.

58. Wright AGC, Zimmermann J. Applied ambulatory assessment: Integrating idiographic and nomothetic principles of measurement. Psychol Assess. 2019 Mar 21. PMID: 30896209. doi: 10.1037/pas0000685.

59. Nock MK, Prinstein MJ. A functional approach to the assessment of self-mutilative behavior. J Consult Clin Psychol. 2004 Oct; 72(5):885-90. PMID: 15482046. doi: 10.1037/0022-006X.72.5.885.

60. Bentley KH, Nock MK, Barlow DH. The Four-Function Model of Nonsuicidal Self-Injury. Clin Psychol Sci. 2014;2(5):638-56. doi: 10.1177/2167702613514563.

61. Kranzler A, Fehling KB, Lindqvist J, Brillante J, Yuan F, Gao X, et al. An Ecological Investigation of the Emotional Context Surrounding Nonsuicidal Self-Injurious Thoughts and Behaviors in Adolescents and Young Adults. Suicide Life Threat Behav. 2018 Apr;48(2):149-59. PMID: 28758704. doi: 10.1111/sltb.12373. 
62. Snir A, Rafaeli E, Gadassi R, Berenson K, Downey G. Explicit and inferred motives for nonsuicidal selfinjurious acts and urges in borderline and avoidant personality disorders. Personal Disord. 2015 Jul;6(3):26777. PMID: 25867834. doi: 10.1037/per0000104.

63. Koenig J, Klier J, Parzer P, Santangelo P, Resch F, Ebner-Priemer U, et al. High-frequency ecological momentary assessment of emotional and interpersonal states preceding and following self-injury in female adolescents. Eur Child Adolesc Psychiatry. 2020 Aug 29. PMID: 32862250. doi: 10.1007/s00787-02001626-0.

64. Voelkle MC, Gische C, Driver CC, Lindenberger U. The Role of Time in the Quest for Understanding Psychological Mechanisms. Multivariate Behav Res. 2018 Nov-Dec;53(6):782-805. PMID: 30668172. doi: 10.1080/00273171.2018.1496813.

65. Burke TA, Hamilton JL, Abramson LY, Alloy LB. Non-suicidal self-injury prospectively predicts interpersonal stressful life events and depressive symptoms among adolescent girls. Psychiatry Res. 2015 Aug 30;228(3):416-24. PMID: 26165966. doi: 10.1016/j.psychres.2015.06.021.

66. Waals L, Baetens I, Rober P, Lewis S, Van Parys H, Goethals ER, et al. The NSSI Family Distress Cascade Theory. Child Adolesc Psychiatry Ment Health. 2018;12:52. PMID: 30568727. doi: 10.1186/s13034-0180259-7.

67. Turner BJ, Cobb RJ, Gratz KL, Chapman AL. The role of interpersonal conflict and perceived social support in nonsuicidal self-injury in daily life. J Abnorm Psychol. 2016 May;125(4):588-98. PMID: 26845256. doi: 10.1037/abn0000141.

68. Taylor PJ, McDonald J, Smith M, Nicholson H, Forrester R. Distinguishing people with current, past, and no history of non-suicidal self-injury: Shame, social comparison, and self-concept integration. J Affect Disord. 2019 Mar 1;246:182-8. PMID: 30583143. doi: 10.1016/j.jad.2018.12.033

69. Burke TA, Piccirillo ML, Moore-Berg SL, Alloy LB, Heimberg RG. The stigmatization of nonsuicidal selfinjury. J Clin Psychol. 2019 Mar;75(3):481-98. PMID: 30368804. doi: 10.1002/jclp.22713.

70. Staniland L, Hasking P, Boyes M, Lewis S. Stigma and nonsuicidal self-injury: Application of a conceptual framework. Stigma and Health. 2020. doi: 10.1037/sah0000257.

71. Nestler S. Modeling intraindividual variability in growth with measurement burst designs. Structural Equation Modeling: A Multidisciplinary Journal. 2020:1-12. doi: 10.1080/10705511.2020.1757455.

62. Buu A, Cai Z, Li R, Wong SW, Lin HC, Su WC, et al. The association between short-term emotion dynamics and cigarette dependence: A comprehensive examination of dynamic measures. Drug Alcohol Depend. 2021 Jan 1;218:108341. PMID: 33268228. doi: 10.1016/j.drugalcdep.2020.108341.

73. Bean CAL, Heggeness LF, Kalmbach DA, Ciesla JA. Ruminative Inertia and Its Association With Current Severity and Lifetime Course of Depression. Clin Psychol Sci. 2020;8(6):1007-16. doi: $10.1177 / 2167702620949174$.

74. Lewis SP, Hasking PA. Self-injury recovery: A person-centered framework. J Clin Psychol. 2020 Dec 9. PMID: 33296508. doi: 10.1002/jclp.23094.

75. Giletta M, Prinstein MJ, Abela JR, Gibb BE, Barrocas AL, Hankin BL. Trajectories of suicide ideation and nonsuicidal self-injury among adolescents in mainland China: Peer predictors, joint development, and risk for suicide attempts. J Consult Clin Psychol. 2015 Apr;83(2):265-79. PMID: 25688812. doi: 10.1037/a0038652.

76. Robinson K, Garisch JA, Wilson MS. Nonsuicidal self-injury thoughts and behavioural characteristics: Associations with suicidal thoughts and behaviours among community adolescents. J Affect Disord 2021. doi: 10.1016/j.jad.2020.12.201.

77. Bos FM, Snippe E, Bruggeman R, Wichers M, van der Krieke L. Insights of Patients and Clinicians on the Promise of the Experience Sampling Method for Psychiatric Care. Psychiatr Serv. 2019 Nov 1;70(11):98391. PMID: 31434558. doi: 10.1176/appi.ps.201900050.

78. Dewa LH, Lavelle M, Pickles K, Kalorkoti C, Jaques J, Pappa S, et al. Young adults' perceptions of using wearables, social media and other technologies to detect worsening mental health: A qualitative study. PLoS One. 2019;14(9):e0222655. PMID: 31532786. doi: 10.1371/journal.pone.0222655.

79. Armey MF. Ecological Momentary Assessment and Intervention in Nonsuicidal Self-Injury: A Novel Approach to Treatment. Journal of Cognitive Psychotherapy. 2012;26(4):299-317. doi: 10.1891/08898391.26.4.299.

80. Arshad U, Farhat Ul A, Gauntlett J, Husain N, Chaudhry N, Taylor PJ. A Systematic Review of the Evidence Supporting Mobile- and Internet-Based Psychological Interventions For Self-Harm. Suicide Life Threat Behav. 2020 Feb;50(1):151-79. PMID: 31448847. doi: 10.1111/sltb.12583.

81. Kruzan KP, Whitlock J, Bazarova NN. Examining the Relationship Between the Use of a Mobile PeerSupport App and Self-Injury Outcomes: Longitudinal Mixed Methods Study. JMIR Ment Health. 2021 Jan 28;8(1):e21854. PMID: 33507154. doi: 10.2196/21854. 
82. Rizvi SL, Hughes CD, Thomas MC. The DBT Coach mobile application as an adjunct to treatment for suicidal and self-injuring individuals with borderline personality disorder: A preliminary evaluation and challenges to client utilization. Psychol Serv. 2016 Nov;13(4):380-8. PMID: 27797571. doi: $10.1037 / \mathrm{ser} 0000100$.

83. Wang L, Miller LC. Just-in-the-Moment Adaptive Interventions (JITAI): A Meta-Analytical Review. Health Commun. 2020 Nov;35(12):1531-44. PMID: 31488002. doi: 10.1080/10410236.2019.1652388.

84. Nahum-Shani I, Smith SN, Spring BJ, Collins LM, Witkiewitz K, Tewari A, et al. Just-in-Time Adaptive Interventions (JITAIs) in Mobile Health: Key Components and Design Principles for Ongoing Health Behavior Support. Ann Behav Med. 2018 May 18;52(6):446-62. PMID: 27663578. doi: 10.1007/s12160016-9830-8.

85. Coppersmith DDL, Dempsey W, Kleiman E, Bentley KH, Murphy SA, Nock MK. Just-in-time adaptive interventions for suicide prevention: Promise, challenges, and future directions. [Preprint: https://psyarxiv.com/eg9fx]. 2021.

86. Liu RT, Sheehan AE, Walsh RFL, Sanzari CM, Cheek SM, Hernandez EM. Prevalence and correlates of non-suicidal self-injury among lesbian, gay, bisexual, and transgender individuals: A systematic review and meta-analysis. Clin Psychol Rev. 2019 Dec;74:101783. PMID: 31734440. doi: 10.1016/j.cpr.2019.101783.

87. Kuentzel JG, Arble E, Boutros N, Chugani D, Barnett D. Nonsuicidal self-injury in an ethnically diverse college sample. Am J Orthopsychiatry. 2012 Jul;82(3):291-7. PMID: 22880967. doi: 10.1111/j.19390025.2012.01167.x.

88. Rad MS, Martingano AJ, Ginges J. Toward a psychology of Homo sapiens: Making psychological science more representative of the human population. Proc Natl Acad Sci U S A. 2018 Nov 6;115(45):11401-5. PMID: 30397114. doi: 10.1073/pnas.1721165115.

89. An S, Cruwys T, Lee H, Chang MX. Cultural Differences in Reactions to Suicidal Ideation: A Mixed Methods Comparison of Korea and Australia. Arch Suicide Res. 2020 Jul-Sep;24(3):415-34. PMID: 31159683. doi: 10.1080/13811118.2019.1624668.

90. Gandhi A, Luyckx K, Adhikari A, Parmar D, Desousa A, Shah N, et al. Non-suicidal self-injury and its association with identity formation in India and Belgium: A cross-cultural case-control study. Transcult Psychiatry. 2020 Sep 2:1363461520933759. PMID: 32873190. doi: 10.1177/1363461520933759.

91. Hunter BH, Radoll PJ. Dynamics of Digital Diffusion and Disadoption: A longitudinal analysis of Indigenous and other Australians. Australasian Journal of Information Systems. 2020;24:1-21

92. Vangeepuram N, Mayer V, Fei K, Hanlen-Rosado E, Andrade C, Wright S, et al. Smartphone ownership and perspectives on health apps among a vulnerable population in East Harlem, New York. Mhealth. 2018;4:31. PMID: 30221166. doi: 10.21037/mhealth.2018.07.02.

93. van Roekel E, Keijsers L, Chung JM. A Review of Current Ambulatory Assessment Studies in Adolescent Samples and Practical Recommendations. J Res Adolesc. 2019 Sep;29(3):560-77. PMID: 31573762. doi: 10.1111/jora.12471.

94. Hasking PA, Lewis SP, Robinson K, Heath NL, Wilson MS. Conducting research on nonsuicidal self-injury in schools: Ethical considerations and recommendations. School Psychology International. 2019;40(3):21734. doi: $10.1177 / 0143034319827056$.

95. Jacobson NC, Bentley KH, Walton A, Wang SB, Fortgang RG, Millner AJ, et al. Ethical dilemmas posed by mobile health and machine learning in psychiatry research. Bull World Health Organ. 2020 Apr 1;98(4):2706. PMID: 32284651. doi: 10.2471/BLT.19.237107.

96. Rintala A, Wampers M, Myin-Germeys I, Viechtbauer W. Response compliance and predictors thereof in studies using the experience sampling method. Psychol Assess. 2019 Feb;31(2):226-35. PMID: 30394762. doi: 10.1037/pas0000662.

97. Nebeker C, Torous J, Bartlett Ellis RJ. Building the case for actionable ethics in digital health research supported by artificial intelligence. BMC Med. 2019 Jul 17;17(1):137. PMID: 31311535. doi: 10.1186/s12916-019-1377-7.

98. Hasking P, Rees CS, Martin G, Quigley J. What happens when you tell someone you self-injure? The effects of disclosing NSSI to adults and peers. BMC Public Health. 2015 Oct 9;15:1039. PMID: 26453187. doi: 10.1186/s12889-015-2383-0.

99. Whitlock J, Lloyd-Richardson E, Fisseha F, Bates T. Parental Secondary Stress: The Often Hidden Consequences of Nonsuicidal Self-Injury in Youth. J Clin Psychol. 2018 Jan;74(1):178-96. PMID: 28493555. doi: 10.1002/jclp.22488.

100. Bai S, Babeva KN, Kim MI, Asarnow JR. Future Directions for Optimizing Clinical Science \& Safety: Ecological Momentary Assessments in Suicide/Self-Harm Research. J Clin Child Adolesc Psychol. 2020 Oct 13:1-13. PMID: 33047987. doi: 10.1080/15374416.2020.1815208.

101. Lewis SP. I cut therefore I am? Avoiding labels in the context of self-injury. Med Humanit. 2017 Sep;43(3):204. PMID: 28264901. doi: 10.1136/medhum-2017-011221. 
102. Hasking P, Boyes M. Cutting Words: A Commentary on Language and Stigma in the Context of Nonsuicidal Self-Injury. J Nerv Ment Dis. 2018 Nov;206(11):829-33. PMID: 30371637. doi: 10.1097/NMD.0000000000000899.

103. Davidson CL, Anestis MD, Gutierrez PM. Ecological Momentary Assessment is a Neglected Methodology in Suicidology. Arch Suicide Res. 2017 Jan 2;21(1):1-11. PMID: 26821811. doi: 10.1080/13811118.2015.1004482.

104. Law MK, Furr RM, Arnold EM, Mneimne M, Jaquett C, Fleeson W. Does assessing suicidality frequently and repeatedly cause harm? A randomized control study. Psychol Assess. 2015 Dec;27(4):1171-81. PMID: 25894705. doi: 10.1037/pas0000118.

105. Selby EA, Kranzler A, Lindqvist J, Fehling KB, Brillante J, Yuan F, et al. The Dynamics of Pain During Nonsuicidal Self-Injury. Clinical Psychological Science. 2018;7(2):302-20. doi: $10.1177 / 2167702618807147$.

106. Husky M, Olie E, Guillaume S, Genty C, Swendsen J, Courtet P. Feasibility and validity of ecological momentary assessment in the investigation of suicide risk. Psychiatry Res. 2014 Dec 15;220(1-2):564-70. PMID: 25155939. doi: 10.1016/j.psychres.2014.08.019.

107. Effect of frequent assessment of suicidal thinking on its incidence and severity: high-resolution real-time monitoring study. The British Journal of Psychiatry. 2021:1-3. doi: 10.1192/bjp.2021.97.

108. Nock MK, Kleiman EM, Abraham M, Bentley KH, Brent DA, Buonopane RJ, et al. Consensus Statement on Ethical \& Safety Practices for Conducting Digital Monitoring Studies with People at Risk of Suicide and Related Behaviors. Psychiatric Research and Clinical Practice. 2020;n/a(n/a). doi: https://doi.org/10.1176/appi.prcp.20200029.

109. Blades CA, Stritzke WGK, Page AC, Brown JD. The benefits and risks of asking research participants about suicide: A meta-analysis of the impact of exposure to suicide-related content. Clin Psychol Rev. 2018 Aug;64:1-12. PMID: 30014862. doi: 10.1016/j.cpr.2018.07.001.

110. DeCou CR, Schumann ME. On the Iatrogenic Risk of Assessing Suicidality: A Meta-Analysis. Suicide Life Threat Behav. 2018 Oct;48(5):531-43. PMID: 28678380. doi: 10.1111/sltb.12368.

111. Muehlenkamp JJ, Swenson LP, Batejan KL, Jarvi SM. Emotional and Behavioral Effects of Participating in an Online Study of Nonsuicidal Self-Injury. Clinical Psychological Science. 2014 Jan;3(1):26-37. PMID: 2015-00515-003. doi: 10.1177/2167702614531579.

112. Cha CB, Glenn JJ, Deming CA, D'Angelo EJ, Hooley JM, Teachman BA, et al. Examining potential iatrogenic effects of viewing suicide and self-injury stimuli. Psychol Assess. 2016 Nov;28(11):1510-5. PMID: 26821197. doi: 10.1037/pas0000280.

113. Hasking P, Tatnell RC, Martin G. Adolescents' reactions to participating in ethically sensitive research: a prospective self-report study. Child Adolesc Psychiatry Ment Health. 2015;9:39. PMID: 26300966. doi: 10.1186/s13034-015-0074-3.

114. Trull TJ, Ebner-Priemer UW. Ambulatory assessment in psychopathology research: A review of recommended reporting guidelines and current practices. J Abnorm Psychol. 2020 Jan;129(1):56-63. PMID: 31868388. doi: 10.1037/abn0000473.

115. Eisele G, Vachon H, Lafit G, Kuppens P, Houben M, Myin-Germeys I, et al. The Effects of Sampling Frequency and Questionnaire Length on Perceived Burden, Compliance, and Careless Responding in Experience Sampling Data in a Student Population. Assessment. 2020 Sep 10:1073191120957102. PMID: 32909448. doi: 10.1177/1073191120957102.

116. Kirtley OJ, Hiekkaranta AP, Kunkels YK, Eisele G, Verhoeven D, Van Nierop M, et al. The experience sampling method (ESM) item repository. 2020. URL: https://doi.org/10.17605/osf.io/kg376.

117. Munafò MR, Nosek BA, Bishop DVM, Button KS, Chambers CD, Percie du Sert N, et al. A manifesto for reproducible science. Nature Human Behaviour. 2017;1(1). PMID: 33954258. doi: 10.1038/s41562-0160021.

118. Kirtley OJ, Lafit G, Achterhof R, Hiekkaranta AP, Myin-Germeys I. Making the Black Box Transparent: A Template and Tutorial for Registration of Studies Using Experience-Sampling Methods. Advances in Methods and Practices in Psychological Science. 2021;4(1). doi: 10.1177/2515245920924686.

119. Horstmann KT. Experience sampling and daily diary studies: Basic concepts, designs, and challenges. The Handbook of Personality Dynamics and Processes2021. p. 791-814.

120. Silvia PJ, Kwapil TR, Walsh MA, Myin-Germeys I. Planned missing-data designs in experience-sampling research: Monte Carlo simulations of efficient designs for assessing within-person constructs. Behav Res Methods. 2014 Mar;46(1):41-54. PMID: 23709167. doi: 10.3758/s13428-013-0353-y.

121. Biddle L, Cooper J, Owen-Smith A, Klineberg E, Bennewith O, Hawton K, et al. Qualitative interviewing with vulnerable populations: individuals' experiences of participating in suicide and self-harm based research. J Affect Disord. 2013 Mar;145(3):356-62. PMID: 23021191. doi: 10.1016/j.jad.2012.08.024. 
122. Littlewood DL, Harris K, Gooding P, Pratt D, Haddock G, Peters S. Using my Demons to Make Good: The Short- and Long-Term Impact of Participating in Suicide-Related Research. Arch Suicide Res. 2019 Sep 23:1-25. PMID: 31544686. doi: 10.1080/13811118.2019.1663330.

123. Kleiman EM, Glenn CR, Liu RT. Real-Time Monitoring of Suicide Risk among Adolescents: Potential Barriers, Possible Solutions, and Future Directions. J Clin Child Adolesc Psychol. 2019 Sep 27:1-13. PMID: 31560584. doi: 10.1080/15374416.2019.1666400.

124. Boden ZV, Gibson S, Owen GJ, Benson O. Feelings and Intersubjectivity in Qualitative Suicide Research. Qual Health Res. 2016 Jul;26(8):1078-90. PMID: 25794522. doi: 10.1177/1049732315576709.

125. Kumar S, Cavallaro L. Researcher Self-Care in Emotionally Demanding Research: A Proposed Conceptual Framework. Qual Health Res. 2018 Mar;28(4):648-58. PMID: 29224510. doi: 10.1177/1049732317746377.

126. Dickson-Swift V, James EL, Kippen S, Liamputtong P. Risk to Researchers in Qualitative Research on Sensitive Topics: Issues and Strategies. Qualitative Health Research. 2008;18(1):133-44. doi: $10.1177 / 1049732307309007$. 
Table 1. Opening the black box of daily life in NSSI research creates new opportunities

1. To better understand the short-term course of NSSI thoughts, urges, and behavior through direct observation and precise measurement.

2. To advance knowledge of individual-level predictors of NSSI thoughts, urges, and behavior and the ability to accurately detect idiographic risk.

3. To test existing theories and develop new models that bridge the idiographic and nomothetic divide and explain who is at risk, and when.

4. To generate insights into the functional mechanisms and relationship of dynamic patterns with dayto-day and meaningful longer-term developmental changes and outcomes.

5. To promote person-centered care and the deployment of personalized prevention and novel digital interventions. 
Table 2. Opening the black box of daily life in NSSI research brings ethical and practical considerations

1. Recruitment should be inclusive from study inception to completion and actively include more vulnerable individuals, with representatives from any vulnerable groups at every stage.

2. The informed consent process should be fully transparent regarding the study demands, the safety protocol, whether data will be passively collected, reimbursement, researchers' responsibility to respond to risk, and potential implications of this responsibility.

3. A proper safety protocol should be developed - with all stakeholders - that matches participants' needs (especially in the event of suicide risk), but that does not inadvertently defeat the study's observational purpose.

4. Although there is no reason to expect that repeated questioning in everyday life will lead to measurement reactivity in NSSI outcomes, researchers are responsible for evaluating whether this holds for all participants in their study.

5. Study designs must be carefully balanced to appropriately answer the research question(s) while not unnecessarily burdening participants. Sufficient resources should be allocated to pilot all aspects of the protocol. Researchers are encouraged to preregister their protocol and be aware of the relevant privacy laws in their home country before commencing data collection.

6. Participants should be recognized as valued research members and receive financial incentives and information about the overall findings. Where feasible, participants should receive feedback on their own data.

7. Research staff should receive good quality training in responding to risk and continued supervision and mentoring. A lone researcher should never be the only person responsible for participants' safety. 


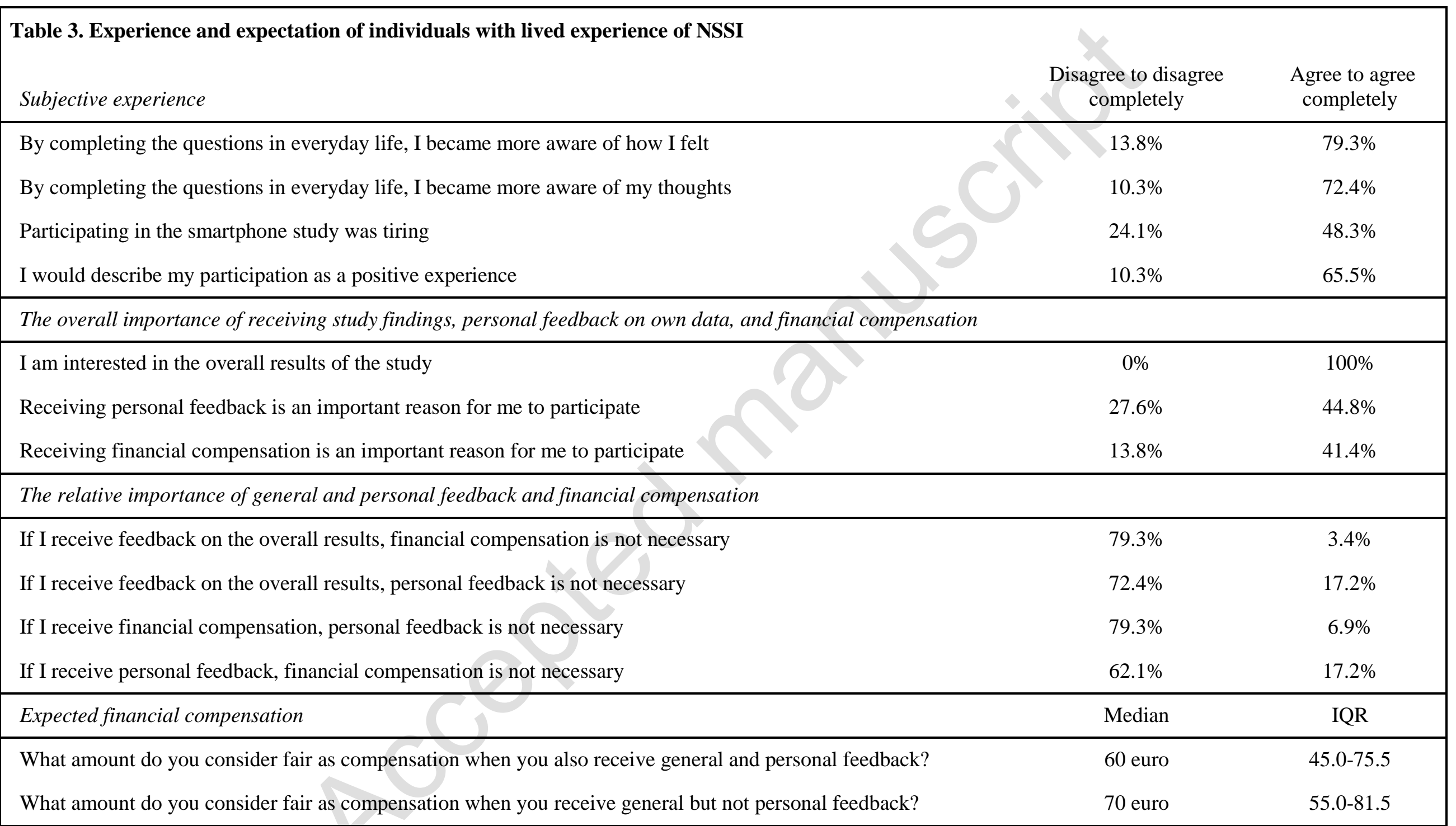

Note: Unpublished data of young adults ( $96.7 \%$ retention, $n=29)$ with lived experience following participation in a 12-day real-time monitoring protocol with 96 semirandom longitudinal assessments (8/day, median compliance 79.2\%; IQR =70.3-91.7\%; Kiekens et al., 2020). The response category 'neutral' is not shown in the table. 


\section{Preparation and planning}

Are measures in place to ensure recruitment will be inclusive to all Adividuals within the target NSSI?

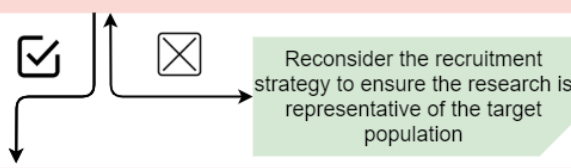

Have representatives of the target population and recruitment site (if applicable) been involved in the design and planning of the real-time monitoring study?

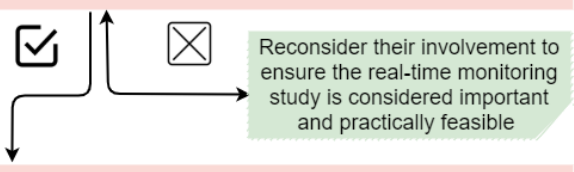

Does the proposed protocol match the research question(s) and has the conceptualization and operationalization been preregistred?

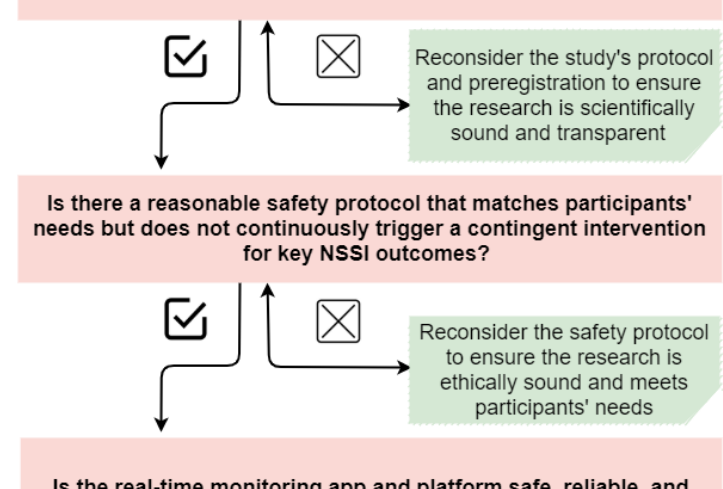
compliant with local privacy and legal regulations?

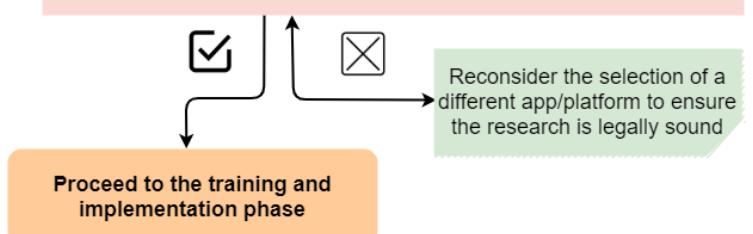

\section{Training and implementation}

Have staff received good quality training to conduct all aspects of and shared responsibility for the study and is there ongoing support and shar
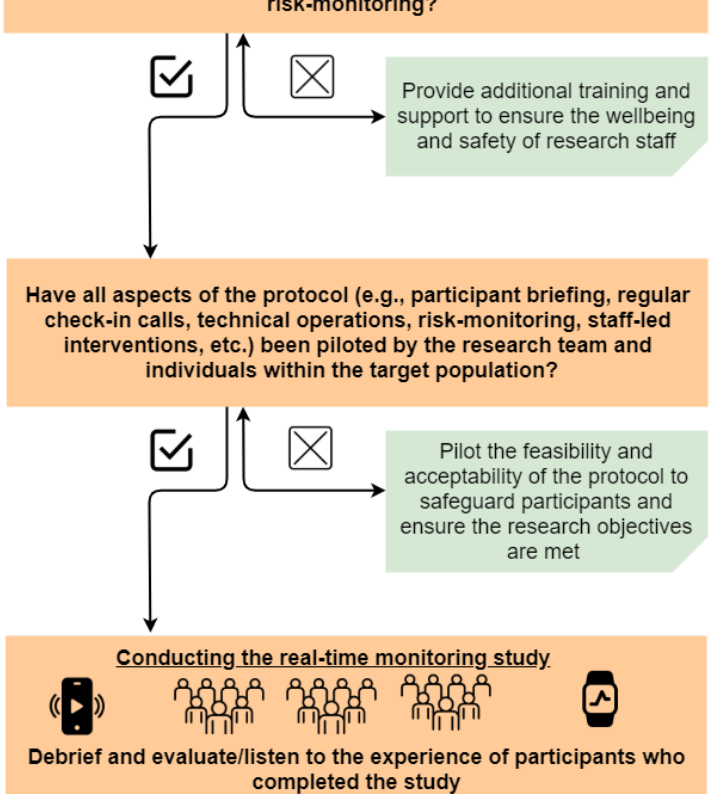

1

Has the necessary sample size/compliance been achieved?

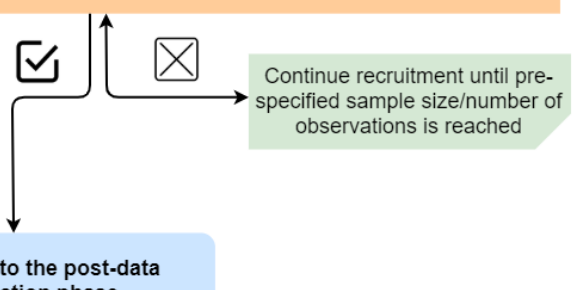

Proceed to the post-data

Figure 1. Flowchart of critical considerations when opening the black box of daily life in NSSI research

\section{Post-data collection}

Have participants (and the recruitment site, if applicable) been recognized as valued members of the research team for their time Provide an overview of the participants

Have all research questions been addressed, psychometrics of NSSI outcomes reported, and findings made publicly available?

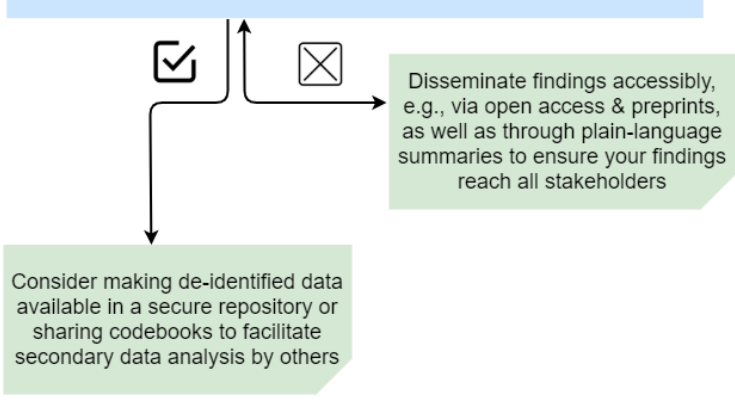

